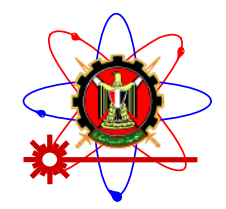

\title{
A Laser Shadowgraphy Methodfor Studying Vibrations of Incandescent Solids
}

\author{
H. S. Ayoub ${ }^{1}$, Ashraf F. El-Sherif ${ }^{2}$, H. H. Hassan ${ }^{3}$, S. A. Khairy ${ }^{4}$ \\ ${ }^{1,3,4}$ Department of Physics, Faculty of Science, Cairo University, Cairo, Egypt \\ ${ }^{2}$ Laser Photonics Research Center, Departmentof EngineeringPhysics, \\ Military Technical College, Cairo, Egypt
}

\begin{abstract}
:
This paper discusses a newlaser shadowgraphymethodfor investigating the in-plane vibration of refractory solid objects at incandescent temperatures.The method is applied whenever the use of Doppler laser vibrometer is inefficient due to small dimensions, high luminosity and poor reflectivity of the test object. We designed an experimental setup that included a modulatedlaser to backlight the vibrating object and to generate an analogue shadowgraph,a photo-detector installed in front of the shadowgraph, isconnected to a lock-in amplifier, and synchronized by the laser modulator,to retrieve the vibration signal from the noisy optical background. The methodwasapplied successfully to detect the resonance frequency of tungsten filament atincandescencetemperature inside an automotive lamp as a proof of concept. This method can be also adapted to test the thermo-elastic characteristics of refractory alloy samples, the mechanical properties of high temperature refractory MEMS lightingsandnano-structured tungsten based advanced thermal shock-resistant lighting sources.
\end{abstract}

\section{Keywords:}

Incandescent temperatures,non-Dopplervibrometry, laser shadowgraphy, low cost method.

\section{Introduction}

Vibration detection is one of the basic needs in experimental physics and mechanical engineering. Perhaps Doppler laser vibrometeris the most important tool used for this purpose[1], also the most fancy and sophisticated piece of equipment that may not be available for many researchers. In spite of its extreme sensitivity, the performance of such equipment is very much dependent on the surface characteristics and emissivity of the object under examination. Incandescent object with high luminosity may not be easy to test fromdistant range in particular cases[2], in addition to the negative effects of laser penetration through the test cell windows that causes beam attenuation, multiple reflections and relative motion readings error. In this work, we tested a tolerated in-plan vibrometry technique based on laser shadowgraphy and synchronous photo-detection, offering a low cost solution that may find its way in manyexperimental fields.For example, it can used to test the mechanical properties of high temperatures refractoryMEMS [3], to investigate the effect of shocks and vibrations on nano-carbon tubes and nano-structured tungsten emitters [4 - 6], in advanced thermal lighting sources.Also it can be used todetect vibration of incandescent materialinducedby photoacoustics[7], where non-contact measurement is needed. This 


\section{Military Technical College \\ Kobry Elkobbah, \\ Cairo, Egypt \\ April 3-5,2018}

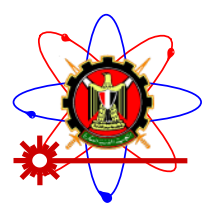

9th International Conference

on Mathematics and

Engineering Physics (ICMEP-9)

technique alsocan be applied without extensive sample preparation as in the case of interferometric detection techniques. Finally, it can be adapted to investigate the mechanical reaction of high energetic materials[8] and solid propellant rods during their combustion. We mention that, the usage incandescent object'sshadowgraph to study its dimensional,positional or vibrational changeshas started at the sixties of the last century. Coaton (1964)[9], designed a setup to investigate the vibration of incandescent lamps based on synchronous stroboscopic illumination of test lamps driven to mechanical resonance using a vibration head. The resonance frequency determination was performed visually by observing the shadow of the filament on a projection screen. In the seventies, Gathers,el al(1976)[10], used a streak camera to record shadowgraphs of metal samples undergoing current pulse heating, to determine their expansivity beyond their melting points. Later, many researchers used shadowgraphy, for monitoring exploding wires [11] and arc welding [12, 13] processes. Finally,Hassan(2015)[14], used laser shadowgraphy of P21W automotive lampto determine the elevated temperature expansivity of non-sag tungsten. In this perspective, we decided to use laser shadowgraphy under synchronous detection detect incandescent object vibration induced by pulse excitation technique.

\section{Theory}

Usually, the object under test in laser shadowgraphy setupsis placed near the focal plane of convex throw lens of focal length $F$, and then back-lighted by laser beam so that, the shadowgraph of the object is project at throw distance $D_{\text {Shadow }}$. The magnification factor $M$ is given by the equation:

$$
M=\frac{D_{\text {Shadow }}-F}{F}
$$

If the object undergoes a small in-plane position shiftdue to vibration, then this shift will be magnified at the shadowgraph plan by a factor of $M$, this technique can detect small shifts as $\delta=1 \times 10^{-7} \mathrm{~m}$, knowing that the theoretical limit[15] is in order of $1 \times 10^{-8} \mathrm{~m}$.

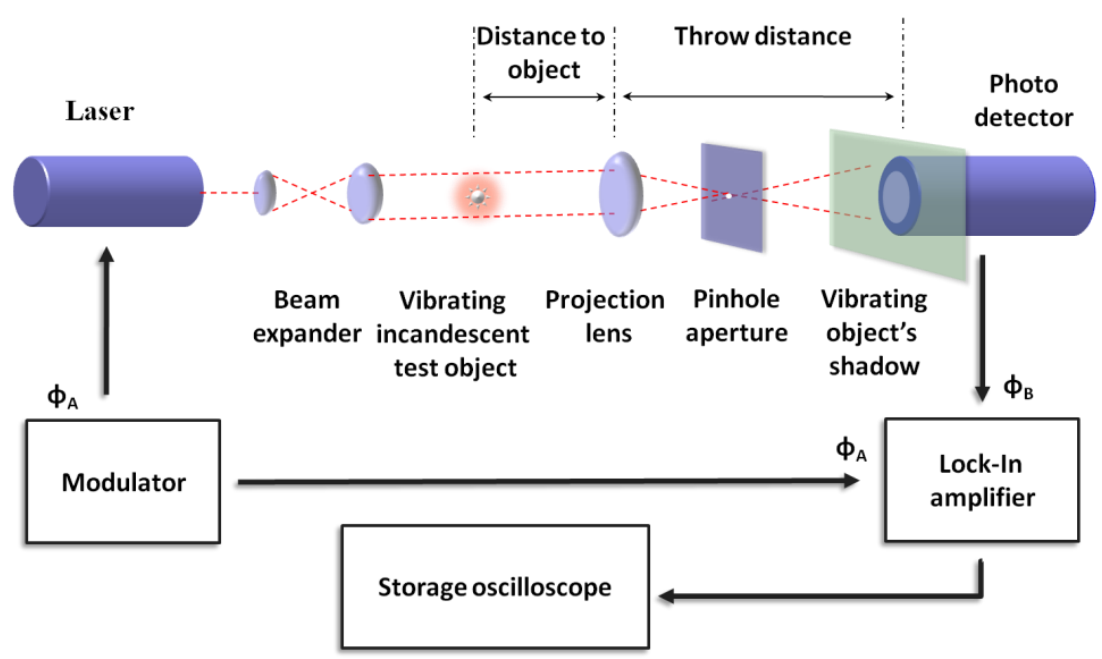


Military Technical College

Kobry Elkobbah,

Cairo, Egypt

April 3-5,2018

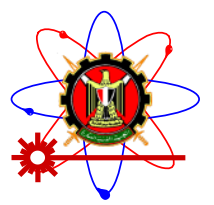

9th International Conference

on Mathematics and

Engineering Physics (ICMEP-9)

Fig.1. Block diagram and basic optics of the shadowgraphy-based vibrometer

In fig.1,the laser beam is modulated by a constant frequency, to allow synchronous detection of time varying optical intensity signal at the shadowgraph plan, caused by the vibration of the object.The amplitude of the lock-in output signalis proportional to the magnitude of laser intensity changes at the object's shadow bright/dark boundaries, or:

$$
I_{\text {Signal }} \propto \Delta I_{\text {laser }}
$$

The luminosity of the object is function of fourth power of its temperature, such that, high intensity incoherent radiationsare emitted from the incandescent object. These radiations can be discarded by using a spatial filter or a pinhole aperture after the throw lens at the focal plane in front of the shadowgraph, by this mean, only laser beam with sharp focus will path through and the incandescent object appears black at the shadowgraph. In order to maximize the output signal, $M$ should kept maximum, the phase angle of the laser reference signal $\varphi_{A}$ and the phase angle photo-detector $\varphi_{B}$ must be in phase and the laser source intensity should be adjusted to bring the photo-detector under optical saturation. In addition, it is important to place the detector's window at the boundary between the object's shadow and the background laser illumination at the shadowgraph plane. Table 1. summarizes the expected basic features and differences between laser shadowgraphy based vibrometry and laser Doppler vibrometry.

Table 1.Basic features of laser shadowgraphy versus laser Doppler vibrometry.

\begin{tabular}{|l|l|l|}
\hline \multicolumn{1}{|c|}{ Parameter } & $\begin{array}{c}\text { Laser shadowgraphy based } \\
\text { vibrometry }\end{array}$ & \multicolumn{1}{|c|}{ Laser Doppler vibrometry } \\
\hline Frequency bandwidth & $\begin{array}{l}\text { Variable (depends on lock- } \\
\text { in time constant ) }\end{array}$ & $\begin{array}{l}\text { Fixed (depends on device } \\
\text { response) }\end{array}$ \\
\hline Optical noise immunity & High & Low \\
\hline Detector saturation & Low & High \\
\hline Vibrometry at incandescent & Easy & Hard \\
\hline Detected phenomena & Laser intensity variations & Laser frequency changes \\
\hline Detected object dimensions & $\begin{array}{l}\text { Suitable for small and micro } \\
\text { structures }\end{array}$ & Suitable for large structures \\
\hline Phenomena causality & Shadow position changes & Radial velocity changes \\
\hline Detected vibration plan & In-plan vibration & Out-of-plan vibration \\
\hline Setup cost & Low & High \\
\hline
\end{tabular}

\section{Experimental setup}




\section{Military Technical College \\ Kobry Elkobbah, \\ Cairo, Egypt \\ April 3-5,2018}

\section{9th International Conference \\ on Mathematics and \\ Engineering Physics (ICMEP-9)}

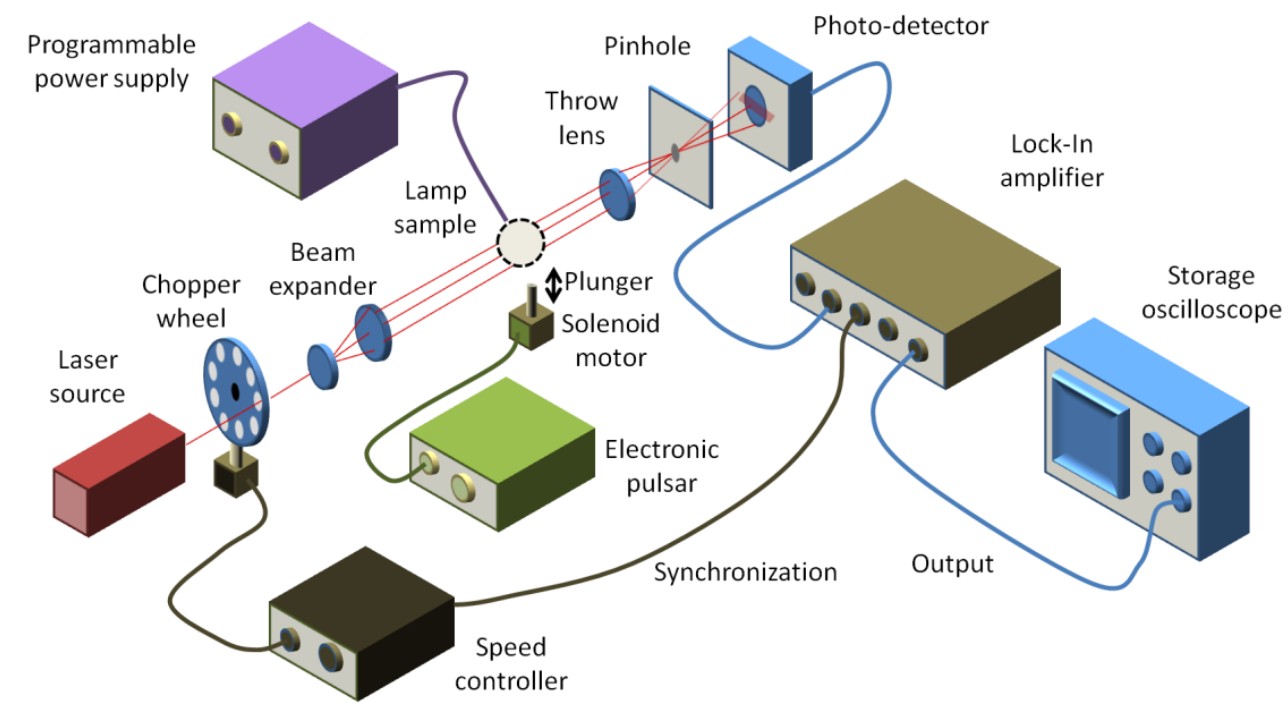

Fig. 2. Illustration of experimental setup used to test laser shadowgraphy-based vibrometer

The designed setup of Fig.2 was realized, using Agilent DSO-X 3052A storage oscilloscope, Scitec 420 Dual Phase lock-in amplifier, 532nm/2W- DPSSL laser diode with modulated power driver(Changchun New Industries Optoelectronics Tech Co.), Agilent 33500B function generator,and DET10C/MThor lab optical detector.

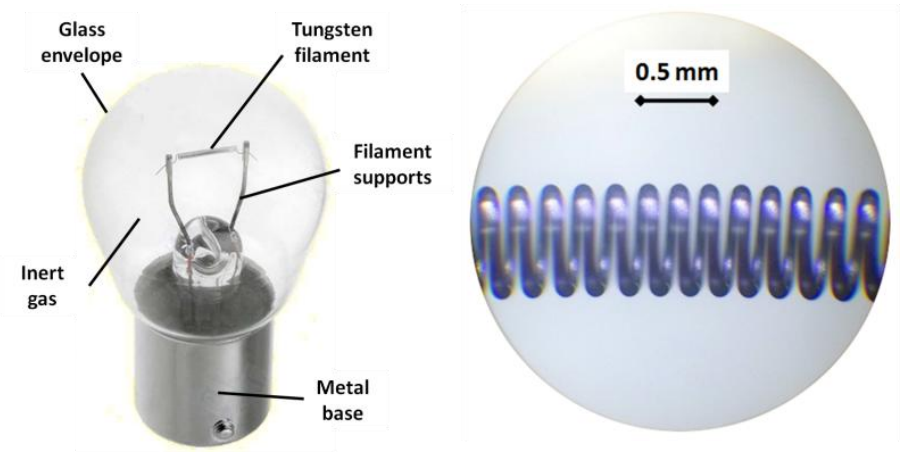

(a) (b)

Fig.2. (a)Tungsten filament of standard P21W automotive lamp used as test object (b)

Traveling microscope image of the tungsten filament

A standard automotive lamp type TARIFA P21W used as incandescent test object (Fig.2). The focal length of the throw lens was $20 \mathrm{~mm}$ and the throw distance was 1 mwith overall magnification of 50x. A small solenoid motor type ZHO-0420L/S, is used to deliver mechanical shocks to the lamp body. 


\section{Military Technical College \\ Kobry Elkobbah, \\ Cairo, Egypt \\ April 3-5,2018}

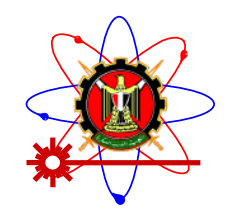

9th International Conference

on Mathematics and

Engineering Physics (ICMEP-9)
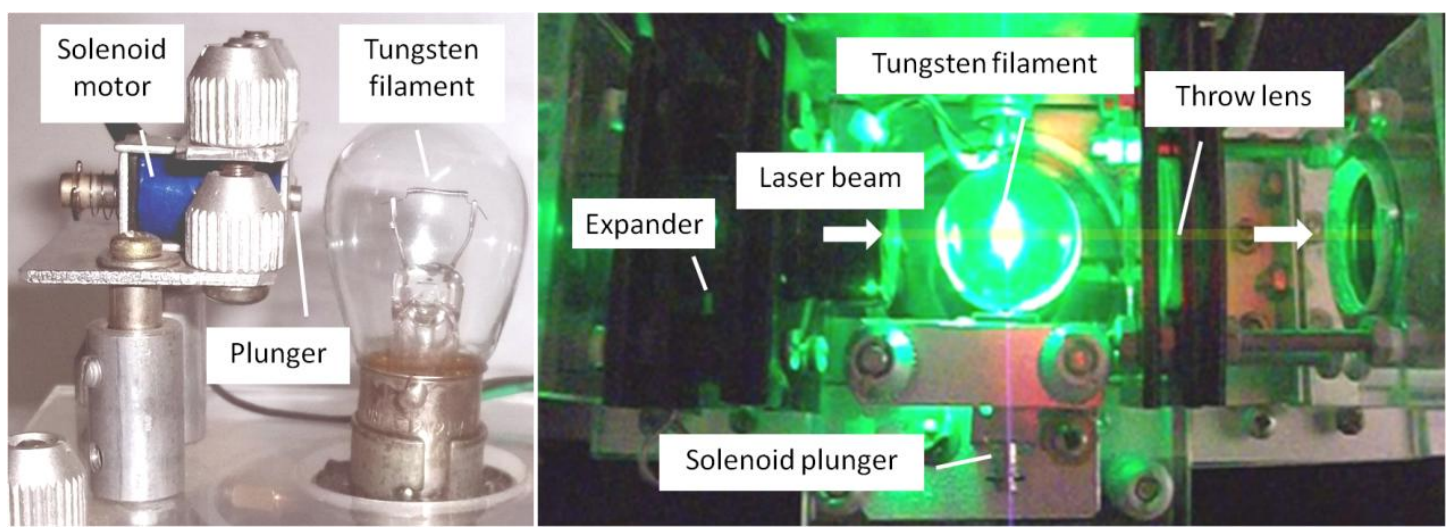

(a) (b)

Fig.3. (a) Tungsten filament lamp used as test objectstroked by a solenoid plunger (b) the sample subject to laser shadowgraphy

Fig.3shows the sample test cell, in which the plunger of the solenoid motor delivers mechanical shocks, to the base fixed lamp body. These shocks induce longitudinal flexure vibration in the tungsten filament. The working temperature of P21W lamp is about 2600 $\mathrm{K}[16]$. The lamp current is increased on incremental base to record the difference in vibration pattern due to the thermal effect on the stiffness of Tungsten filament. The laser modulation frequencymust chosen to be at least 10 times the expected resonance frequency of the filament. The prediction of flexure frequency of the filament is possible,by applying the equation of natural frequency $f$ of helical compression spring[17]:

$$
f=\frac{d}{9 N D^{2}} \sqrt{\frac{G}{\rho}}
$$

Where $D$ is the filament coil diameter, $d$ is the coil wire diameter, $\rho$ is the wire material density, $n$ is the number of coil windings and $G$ is the dynamic shear modulus of the coil material. For the tested samples $d=80 \times 10^{-6} \mathrm{~m}, D=0.57 \times 10^{-3} \mathrm{~m}, \mathrm{~N}=150, G=290 \mathrm{GPa}[18]$, $\rho=19300 \mathrm{Kg} / \mathrm{m}^{3}$, so the natural frequency of the filament is approximatelynear700 Hzmaking the laser modulation frequency to be around $10 \mathrm{kHz}$.

\section{Results and Discussions}

The obtained shadowgraph of the incandescent filament was enhanced after the addition of pinhole filter, to discard the yellowishluminanceof the sample, and to avoid saturation of the photo-detector (as shown in Fig.4). 


\section{Military Technical College \\ Kobry Elkobbah, \\ Cairo, Egypt \\ April 3-5,2018}

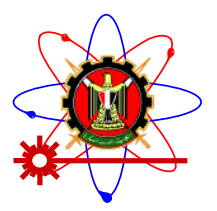

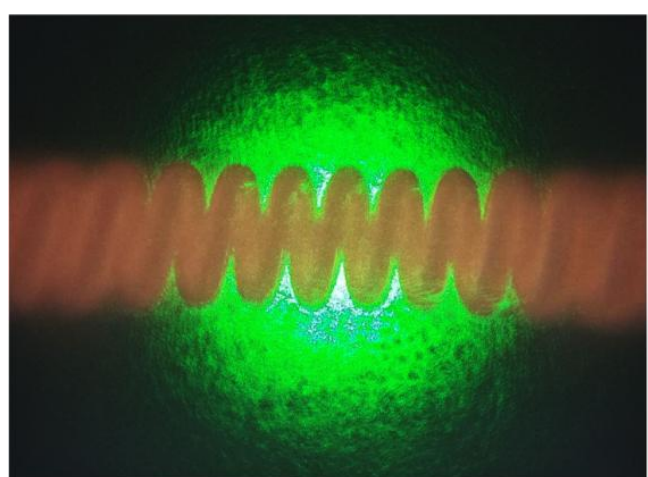

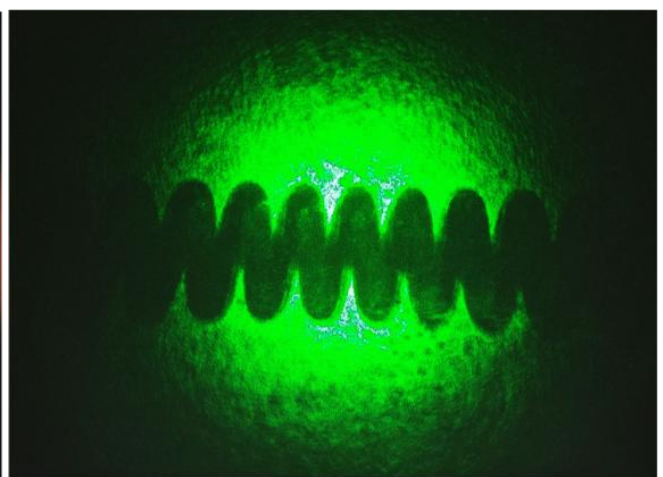

(a) (b)

Fig.4. Laser shadowgraph of the tungsten filament at incandescence, a) without pinhole filter, b)with pinhole filter

Under successive shocks delivered by the solenoid motor plunger, to the lamp envelope along the central axe of the filament, the lock-in amplifier detects the weak AC signal, of the inplane vibration of the sample's shadowgraph.As shown in Figure (5), the resultant vibration pattern shows pulse activated decayed resonance frequency of around $700 \mathrm{~Hz}$ as approximated by equation 3 .
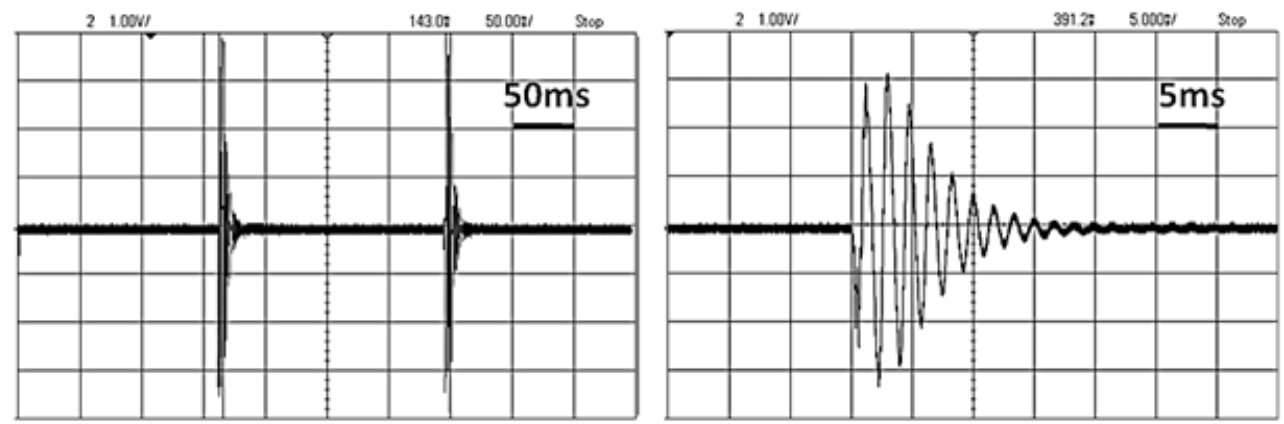

(a) (b)

Fig.5.Shock induced in-plan longitudinal vibration of tungsten filament a) repetitive pulse resonance b) single resonance pattern

The vibration pattern of the incandescent filament shows a noticeable difference as the lamp is turned on and off (as seen in Fig.6).
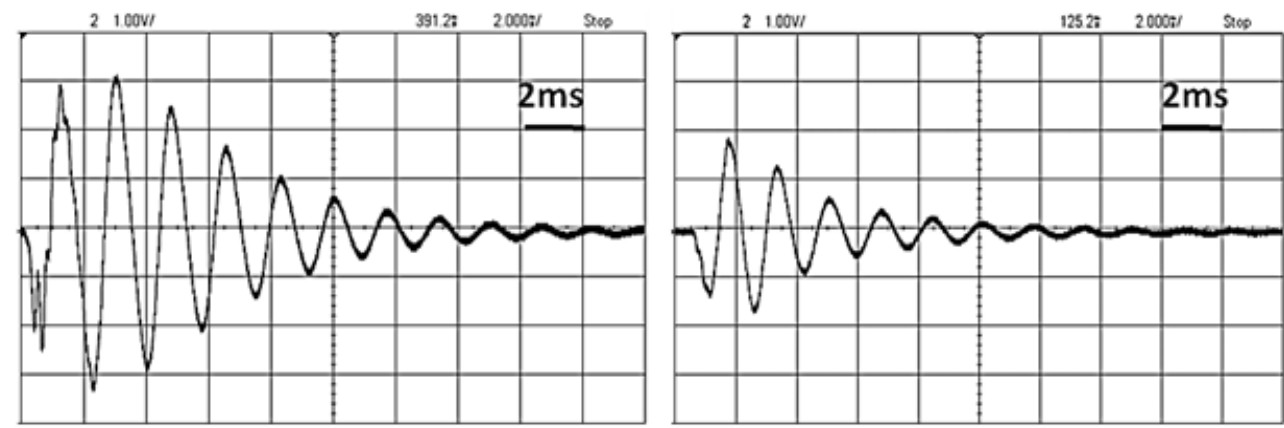
Military Technical College

Kobry Elkobbah,

Cairo, Egypt

April 3-5,2018

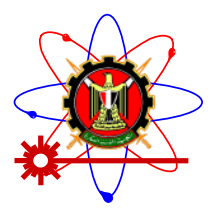

(a) (b) 9th International Conference

on Mathematics and

Engineering Physics (ICMEP-9)

Fig.6.Longitudinalvibration amplitude of tungsten filament, a) at room temperature,

b) at $2600 \mathrm{~K}$

Thedecreasein the quality factor of the damped vibrationamplitude in the case where the filament is hot,is due to the change in internal friction of the tungsten with the increase of temperature[19]. The test was repeated at different filament temperatures, by changing the filament supply current. The temperature $T$ of the filament was calculated from the equation[20]:

$$
T=T_{o}\left(\frac{R}{R_{o}}\right)^{0.83}
$$

Where $T_{o}=300 \mathrm{~K}, R_{o}$ is thefilament resistance at $300 \mathrm{Km}$ masured by ohmmeter, and $R$ is the filament resistance at temperature $T$, obtained from the voltage-current data.A decrease of the vibration frequency of the filament as the temperature increases was observed (as shown at Fig.7.) This changeis due to the decrease in the shear modulus Gof tungsten (according to eq.3) as result of temperature change[19].

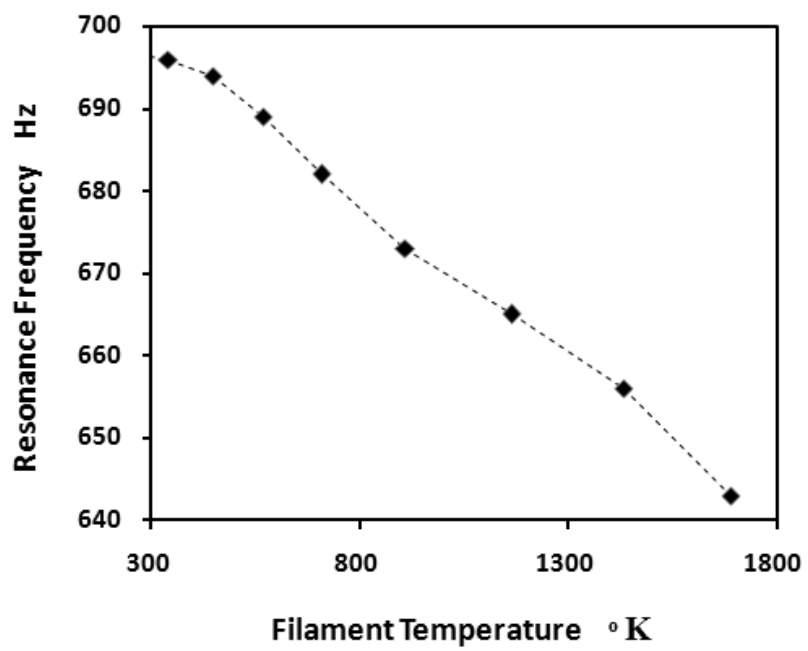

Fig.7Longitudinalvibration frequency of the tungsten filament versus temperature

The polynomial regressionof the previous data yielded a parametric equation describing the frequency of the tested filament as a function of temperature on the form:

$$
\boldsymbol{f}=7 \times 10^{2}-4.42 \times 10^{-2} \boldsymbol{T}+2.86 \times 10^{-4} \boldsymbol{T}^{2}-8.18 \times 10^{-7} \boldsymbol{T}^{3}+9.2 \times 10^{-10} \boldsymbol{T}^{4}-4.59 \times 10^{-13} \boldsymbol{T}^{5}(5)
$$

This equation is valid for temperatures in the range of $300-2000 \mathrm{~K}$. The example of the tungsten filament vibration, reveals the capability of shadow vibrometry technique to perform accurate remote measurements at elevated temperatures with a relatively simple setup.

\section{Conclusion}




\section{Military Technical College \\ Kobry Elkobbah, \\ Cairo, Egypt \\ April 3-5,2018}

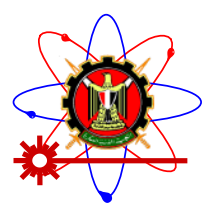

Thesynchronous non-Dopplerlaser shadowography based vibrometrytechnique has successfully proven very effective as a vibration detection tool, for elevated temperature incandescent objects.It is non-contact, low noise, simple and low cost. It does not need extensive sample preparation, because it does not dependson the surface characteristics of the sample neither their radiant emissions nor their dimensions. It can hold all information about the in-plane vibration of thesample without attenuation or damping. Therefore, it is a promising tool in many fields of high temperature technology. The expected application of such method is, monitoring the vibrations of shock-resistant thermal lighting,testingthe thermo-elastic characteristics of refractory alloy samples, the mechanical properties of high temperature refractory MEMSmicro lightingsand nano-structured tungsten based advanced thermallighting sources.

\section{Acknowledgment}

The authors are very grateful to the members ofPhysics Department, Faculty Science, Cairo University, Laser Research Technology Center, Engineering Physics Department, Military Technical College for their support with measurement instruments, encouragement andhelpful suggestions.

\section{References}

[1] P. Castellini, M. Martarelli, E.P. Tomasini, Laser Doppler Vibrometry: Development Of Advanced Solutions Answering to Technology's needs, Mechanical systems and Signal Processing 20, 1265-1285, 2006. [2] J. Vass, R. Smid, R.B. Randall, P. Sovka, C. Cristalli, B. Torcianti, Avoidance of speckle noise in laser Vibrometry by the use of kurtosis ratio: Application to mechanical fault diagnostics, Mechanical Systems and Signal Processing 22, 647-671, 2008.

[3] G. Rehder, M.N.P. Carreno, M.I. Alayo, Simple MEMS-based Incandescent Microlamps, ECS Transaction, 9(1), 489-496, 2007.

[4] M.I. Alayo, G. Rehder, and M.N.P. Carreno, MEMS-based incandescent microlamps for integrated optics applications, Journal of pure applied optics 10 (10), 104022, 2008.

[5] Jinquan Wei, Hongwei Zhu, Dehai Wu, Bingqing Wei, Carbon nanotube filaments in household light bulbs, Applied Physics Letters, 24(84), 4869-4871, 2004.

[6] A.Y. Vorobyev, V.S. Makin, ChunleiGoe, Brighter light sources from black metal: Significant increase in emission efficiency of incandescent light sources, Physical Review Letters (102), 234301, 2009.

[7] Jean-Pierre Monchalin, Jean-Daniel Aussel, Paul Bouchard and Rene Heon,LASER-ULTRASONICS FOR INDUSTRIAL APPLICATIONS, Porceeding of Industrial Review of Progress in Quantititive

Nondestructive Evaluation NDE, D. O. Thompson and D.E. Chimemti, Eds. New York: Plenum Vol. 7B, $1607-$ 1614,1988

[8] M.M. Biss, G.S. Settles, M.J. Hargather, L.J. Dodson, and J.D. Miller, High-speed digital shadowgraphy of shock waves from explosions and gunshots, Shock Waves, Springer Book, 91-96, 2009. [9] J. R. Coaton, Vibration in filament lamps, A.E.I. Lamp and Lighting Company, UK, 32 (128), 195 - 200, 1962.

[10] G. R. Gathers, J. W. Shaner and R. L. Brier, Improved apparatus for thermophysical measurements on liquid metals up to 8000K, Rev. Sci. Instrum. 47(471), 1976.

[11] Deeney, C., McGurn, J., Noack, D., Porter, J. L., Spielman, R. B., M. R., Matzen, M. K, Streaked Laser Shadowgraphy Of Tungsten Wire Array Implosions On The Saturn Generator, review Of Scientific Instruments, 68(1), 647, 1997.

[12] S. Subramaniamy, D R White, D J Scholl, W H Weber, In situ optical measurement of liquid drop surface tension in gas metal arc welding, J. Phys. D: Appl. Phys., 31, 1963-1967, 1998. 
Military Technical College

Kobry Elkobbah,

Cairo, Egypt

April 3-5,2018

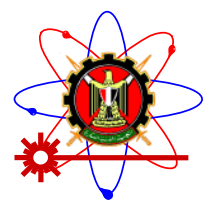

9th International Conference

on Mathematics and

Engineering Physics (ICMEP-9)

[13] C. C. Rusu, L. R. Mistodie, and E. Ghita, Laser Shadowgraph System For The Electrical Arc Investigation, U.P.B. Sci. Bull., Series D, 73(2), 2011.

[14] H.H. Hassan, S.A. Khairy and H.S. Ayoub, A Simple Laboratory Experiment for Measuring the Expansivity of Tungsten at Elevated Temperatures, Nature and Science; 13(11), 146-151, 2015.

[15] E. G. Wolff, Measurement Techniques for Low Expansion Materials, 9th National SAMPE Technical Conference, AtlantaGa, 9, 57-72, 1977.

[16] C. Izarra and J. M. Gitton, Calibration And Temperature Profile Of A Tungsten Filament Lamp, European Journal of Physics, 2010.

[17] A. M. Wahl, Mechanical Springs, 2nd Edition, McGraw-Hill, Inc., New York, 1963.

[18] Igor L. Shabalin, Ultra-High Temperature Materials I: Carbon (Graphene/Graphite) and refractory metals, springer science + business media Dordrechet, ISBN 978-94-007-7586-2, 2014.

[19] I. BERLEC, "The effect of impurities and heat treatment on the internal on the internal friction of Tungsten at high temperatures", Metallurgical Transactions, 1, 2677-2683, 1970.

[20] D. C. Agrawal, V. J. Menon, "Light bulb exponent-rules for the classroom", IEEE Transactions on Education, 43, 262-265, 2000.

\section{Highlights}

\section{Our research work features:}

1. New laser shadowgraphy method to detect vibration of incandescent solids.

2. Vibration signal is retrieved by synchronous photo-detection of shadow intensity

3.Measurements are independent on sample reflectivity, luminosity or dimensions.

4. Low cost, non contact, sensitive and accurate vibrometry method.

5. Suitable for micro MEMS thermal lighting sources vibrometry and testing. 